\title{
Dynamics of Protein-Surfactant Exchange at the Air-Water Interface Studied by Optical Second Harmonic Generation and Ellipsometry
}

\author{
S. W. H. Eijt, M. M. Wittebrood, M. A. C. Devillers, and Th. Rasing* \\ Research Institute for Materials, University of Nijmegen, Toernooiveld, \\ NL-6525 ED Nijmegen, The Netherlands
}

Received May 10, 1994. In Final Form: September 2, $1994^{\otimes}$

\begin{abstract}
The exchange behavior between proteins and surfactants at the water-air interface has been studied using optical second harmonic generation and ellipsometry. At low surfactant concentrations the proteins force the adsorbed surfactants to dissolve, whereas at higher concentrations a double layer is formed with the surfactants on top of the proteins.
\end{abstract}

\section{Introduction}

The properties of proteins at a water surface have acquired a lot of attention due to their important role in understanding certain biological processes. ${ }^{1,2}$ One of the most interesting topics in this field is concerned with the exchange behavior of proteins and other, usually small, molecules. ${ }^{3}$ In this way the permeability of protein layers and other basic problems concerning the adsorption competition between different kinds of molecules can be studied. Further, knowledge of exchange processes of proteins is important for industrial applications, for example in understanding the effect when detergents or soaps for cleaning procedures are supplied. ${ }^{4}$

Therefore, research in this area has been numerous. However, the experimental techniques most commonly used, like surface tension or surface potential measurements, each have their limitations. Surface tension or surface potential is not directly related to the density of the molecules present at the surface, and they require a specific knowledge of pressure-area characteristics. ${ }^{5}$

In this article, we used optical second harmonic generation (SHG), a now well-established technique on studies of adsorbates and interfaces. ${ }^{6,7}$ Further, ellipsometry is used as a complementary tool, as it also has monolayer sensitivity. ${ }^{8}$ SHG is a nondestructive in situ probe, capable of determining adsorbate surface densities and (average) molecular orientation, without requiring knowledge of surface pressure $p$ or temperature $T$. Its sensitivity is based on the symmetry breaking at interfaces. Further, SHG is capable of covering a wide range of concentrations. ${ }^{9}$ As it turns out, the SHG signal is dominated by the surfactant response, whereas the proteins are mostly responsible for the ellipsometry

${ }^{\otimes}$ Abstract published in Advance ACS Abstracts, November 1, 1994.

(1) Gaines, G. L., Jr. Insoluble Monolayers at Liquid-Gas Interfaces; Interscience Publ.: New York, 1966.

(2) Adamson, A. W. Physical Chemistry of Surfaces, 2nd ed.; Interscience Publ.: New York, 1967.

(3) Schulman, J. H.; Hughes, A. H. Proc. R. Soc. (London) 1937, B122, 29 .

(4) Rosen, M. J. Surfactants and Interfacial Phenomena, 2nd ed.; J. Wiley and Sons: New York, 1989.

(5) Langmuir, I.; Schaeffer, V. J. J. Am. Chem. Soc. 1937, 59, 2400.

(6) Rasing, Th.; Stehlin, T.; Shen, Y. R.; Kim, M. W.; Valint, P., Jr. J. Chem. Phys. 1988, 89, 3386. Grubb, S. G.; Kim, M. W.; Rasing, Th.; Shen, Y. R. Langmuir $1988,4,452$.

(7) Shen, Y. R. Nature 1989, 337, 519. Vogel, V.; Shen, Y. R. Annu. Mater. Sci. 1991, 21, 515.

(8) Rasing, Th.; Hsuing, H.; Shen, Y. R.; Kim, M. W. Phys. Rev. A $1988,37,2732$

(9) Bhattacharyya, K.; Castro, A.; Sitzmann, E. V.; Eisenthal, K. B. J. Chem. Phys. 1988, 89, 3376.

0743-7463/94/2410-4498\$04.50/0 signals. We will show that by combining both techniques the adsorption kinetics and energetics and the dynamics of the exchange interactions of the mixed proteinsurfactant system can be studied quantitatively in this way. The results show that the final equilibrium positions depend on the concentrations of the molecules involved. They also show the power of these combined optical techniques for the study of interfacial phenomena, which is of interest not only for biological systems but also for polymer exchange dynamics at interfaces.

The SHG response of an adsorbate-surface system can schematically be written as

$$
I(2 \omega) \propto\left|\chi_{\mathrm{s}}^{(2)}+\chi_{\mathrm{m}}^{(2)}\right|^{2} I^{2}(\omega)
$$

with $\chi_{s}^{(2)}$ the nonlinear susceptibility of the surface and $\chi_{\mathrm{m}}^{(2)}$ the monolayer response (a possible interaction term between the monolayer and the surface has been ignored here). The latter can be written as

$$
\chi_{\mathrm{m}, j j k}^{(2)}=N_{\mathrm{s}}\left\langle T_{i j k}^{\xi \eta \xi}\right\rangle \alpha_{\xi \eta \xi}^{(2)}
$$

with $N_{\mathrm{s}}$ the molecular density, $T_{i j k}^{\xi \eta \xi}$ the transformation between the molecular and laboratory coordinates, and $\alpha_{\xi \eta \zeta}^{(2)}$ the molecular nonlinearity. The brackets indicate an average over the molecular orientations. Equations 1 and 2 show how SHG can be used to probe the molecular density and orientation at the surface. Therefore SHG can be used as an in situ probe to monitor the dynamics of surface active molecules. ${ }^{6}$ Another advantage is its spectroscopic ability to discriminate between different kinds of molecules, though generally for this, sum frequency generation offers better possibilities. ${ }^{10}$

The system investigated here was the exchange of proteins and surfactants at the water-air interface. Discrimination between the two kinds of molecules was based upon the detectable nonlinear optical response of the surfactants, in contrast to the very low SHG response of the proteins.

Contrary, the data obtained by ellipsometry reflected to a large extent the behavior of the proteins. Two parameters, $\Delta$ and $\Psi$, are determined by this technique from the intensity and polarization of the incident and reflected fundamental beams ${ }^{11}$ following

(10) Guyot-Sionnest, P.; Hunt, J. H.; Shen, Y. R. Phys. Rev. Lett. 1987, 59, 1597

(11) Rasing, Th.; Shen, Y. R.; Kim, M. W.; Grubb, S. G. Phys. Rev. Lett. 1985, 55, 2903. 


$$
\varrho=\frac{r_{\mathrm{p}}}{r_{\mathrm{s}}}=\tan \Psi \mathrm{e}^{\mathrm{i} \Delta}
$$

where $r_{\mathrm{p}}$ and $r_{\mathrm{s}}$ are the linear Fresnel coefficients for $\mathrm{p}$ and s-polarized light, respectively. For a thin interface layer model $\left(n_{1}^{\text {ads }} d \ll \lambda\right)$ the change in these parameters $\delta \Delta$ and $\delta \Psi$ are related to the real and imaginary parts of the dielectric constant of this layer:

$$
\begin{gathered}
\delta \Delta \propto \Delta n_{1} d \\
\delta \Psi \propto n_{2} d
\end{gathered}
$$

where $\Delta n_{1}=n_{1}^{\text {ads }}-n_{1}^{\text {water }}$ and $n_{2}=n_{2}^{\text {ads }}$. The dielectric properties of the thin adsorbed layer are given by $n^{\text {ads }}=$ $n_{1}^{\text {ads }}+i n_{2}^{\text {ads }}$. In order to calculate $\Delta n_{1} d$ and $n_{2} d$ from the measured parameters, a three-phase model is used. ${ }^{12}$

\section{Experiment}

The protein used is a lipase from the thermophilic fungus Humicola lanuginosa. The natural substrate for lipases are triglycerides. Fatty acyl ester bonds are cleaved from the glycerol backbone by this fat-degrading enzyme. This rather small protein $\left(M_{\mathrm{w}} \approx 30000 \mathrm{~g} / \mathrm{mol}\right.$ ) was selected, expecting to cover a relatively small area per molecule at the surface compared to other usually larger proteins. The proteins themselves appeared to have a very small SHG response. This can be understood, because per protein molecule there are only four tryptophane side groups that can be expected to give an appreciable nonlinear response.

The surfactant molecule used is Antarox CO 630 (Rhône Poulenc) ( $p$-nonylbenzene nonaethylene oxide). This molecule belongs to a well-studied group of detergents, the POEs (poly(oxyethylenated) alkylphenols). ${ }^{4}$ Its molar weight is $580 \mathrm{~g} / \mathrm{mol}$. For the SHG experiments, the benzene ring is expected to give the major contribution to the nonlinear signal. ${ }^{13,14}$ Its adsorption behavior as detected by SHG therefore can be directly compared to surface tension measurements performed on similar molecules. ${ }^{15,16}$

The excitation light source was a copper vapor laser at 511 $\mathrm{nm}$. Typical peak power levels on the water surface were 6.5 $M W / \mathrm{cm}^{2}$ in $12 \mathrm{~ns}$ pulses at a repetition frequency of $6.5 \mathrm{kHz}$. The beam was focused to a diameter of about $0.36 \mathrm{~mm}$. The weak SHG beam was separated from the strong fundamental reflection by color filters.

As only intensity information was important, unpolarized input beams and SHG beams were used in the exchange experiments. The intensity was measured using a gated photon counting detection scheme.

For the ellipsometry measurements a Gaertner $\mathrm{L} 117 \mathrm{C}$ manual ellipsometry setup was used, with the light beam of a HeNe laser at $632.8 \mathrm{~nm}$ in near Brewster angle geometry (angle of incidence $\left.\phi_{\mathrm{i}}=55^{\circ}\right)$. The beam diameter was $1 \mathrm{~mm}^{2}$.

In each of the experiments the same amount of proteins, $2 \times$ $10^{17}$ molecules, was supplied to a $59 \mathrm{~mL}$ distilled water filled glass trough, with an area of $69 \mathrm{~cm}^{2}$. It should be noted that the protein samples were stored at room temperature. The distilled water solutions used in the experiment where freshly prepared in each case. However, this approach might result in a denaturation of the proteins. Since in this experiment the main point of interest is the study of the interaction between surfactants and proteins at an interface, eventually occurring (reproducable) changes in the protein shape are not relevant. The Antarox concentrations in aqueous solution as used in the supplying

(12) Azzam, R. M. A; Bashara, N. M. Ellipsometry and Polarized Light; North Holland: Amsterdam, 1987.

(13) Rasing, Th.; Berkovic, G.; Shen, Y. R.; Grubb, S. G.; Kim, M. W. Chem. Phys. Lett. 1986, 130, 1 .

(14) Berkovic, G.; Rasing Th., Shen, Y. R. J. Opt. Soc. Am. B 1987, 4,945 .

(15) Crook, E. H.; Fordyce, D. B.; Trebbi, G. F. J. Phys. Chem. 1963, $67,1987$.

(16) Crook, E. H.; Trebbi, G. F.; Fordyce, D. B. J. Phys. Chem. 1964, 68,3592 . pipette or tube were calibrated with the intensity of the integrated absorption peak in the interval 265-285 $\mathrm{nm}$. Before every measurement, the trough and supplying equipment were thoroughly cleaned in an ultrasonic bath using demiwater and rinsed by a $40 \%$ sulfuric acid solution.

\section{Results and Discussion}

First the adsorption kinetics of the surfactants was studied. In this experiment an Antarox monolayer (reaching approximately $85 \%$ of the saturation density using $C_{\text {bulk }}=2 \times 10^{-6} \mathrm{M}$ ) was supplied from above by a pipette to the neat water solution to form a stable homogeneous layer, usually within a few minutes. The Antarox layer appeared to be stable in the detectable region (above approximately 0.2 of a monolayer), as judged by the second harmonic signal. The square root of the SHG signal, proportional to the surface density $N_{\mathrm{s}}$, shows a nice Langmuir adsorption behavior as indicated by the solid line (see Figure 1). From the stability and reproducibility of the signals we can conclude that the surfactants form a homogeneous layer, or in the case of island formation, the islands must be a lot smaller than the beam size $\left(0.1 \mathrm{~mm}^{2}\right)$, so that the experiment averages over these islands.

The surface density in this model is related to the bulk free surfactant concentration by the following: ${ }^{5}$

$$
\frac{N_{\mathrm{s}}}{N_{\mathrm{sat}}}=\frac{C_{\text {bulk }}}{C_{\text {bulk }}+K}
$$

where $N_{\mathrm{s}}$ and $N_{\text {sat }}$ are the surface molecular densities present at bulk concentration $C_{\text {bulk }}$ and saturation, respectively. The adsorption constant $K$ is related to the adsorption free energy $\Delta G$ by $K / 55.3=\exp (\Delta G / R T) .{ }^{4}$ From Figure $1 \Delta G$ is estimated to be $-46.1 \pm 0.3 \mathrm{~kJ} / \mathrm{mol}$, in good agreement with free energies obtained by surface tension measurements on similar molecules. ${ }^{15,16}$ At variance with this is the observation that the monolayer coverage is obtained for a bulk concentration of $\sim 10^{-5} \mathrm{M}$, i.e. an order of magnitude below the cmc value of $3 \times 10^{-4} \mathrm{M}$, as obtained from surface tension measurements. The reason for this observation is so far unclear to us. At the monolayer coverage (as observed from SHG), the area per molecule is $54 \pm 7 \AA^{2}$.

Because a pure protein monolayer showed no effect on the SHG response of the water surface, the results of Figure 1 can be used as a calibration of the Antarox surface density for the mixed systems.

The SHG response of the surfactant-water system appears to be much smaller when the surfactants are supplied from below the interface, that is directly into the water using a tube. The observed intensity in this case is depicted in Figure 1 by the open circles. There was no indication of kinetical limitations, as these signals remained stable in time. For low concentrations the signal level decreases to even below $20 \%$ of the expected value. This effect is related to the known formation of micelles of the surfactants above a certain critical concentration, the cmc value. In supplying the surfactants, concentrations of the order of $10^{-3} \mathrm{M}$ were used in aqueous solutions. Therefore, when the surfactants are supplied smoothly to the solution by means of a tube, initially micelles will be present in the solution. The effective free surfactant bulk concentration is diminished, and correspondingly, according to eq 6 , the surface density is lower. When the surfactants are deposited from above, as in the first case using a pipet, the micelles seem to break up as indicated by the filled circles in Figure 1. This indicates the presence of an energy barrier against the breaking up of the micelles. 


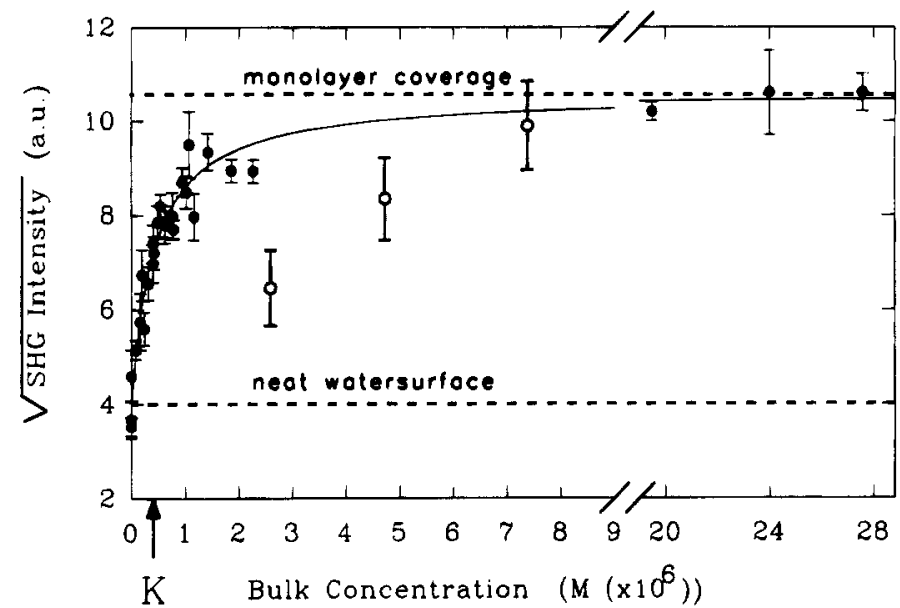

Figure 1. Square root of the second harmonic intensity versus the bulk concentration of an aqueous Antarox solution at $20^{\circ} \mathrm{C}$. The solid line is a theoretical curve representing the best fit according to a Langmuir adsorption behavior. It is characterized by the bulk concentration $\mathrm{K}=(0.36 \pm 0.05) \times 10^{-6} \mathrm{M}$. The open circles are measured when the surfactants are delivered underneath the interface.

Ellipsometry appeared to be more sensitive to the protein layer than to the surfactant layer. For the two cases we found

$$
\begin{gathered}
\delta \Delta_{\text {protein }}=(11.0 \pm 0.2)^{\circ} ; \delta \Psi_{\text {protein }}=(0.0 \pm 0.2)^{\circ} \text { and } \\
\delta \Delta_{\text {surf }}=(2.0 \pm 0.2)^{\circ} ; \delta \Psi_{\text {surf }}=(0.0 \pm 0.2)^{\circ}
\end{gathered}
$$

The values for $\delta \Delta$ correspond in the three-layer model to

$$
\begin{gathered}
\Delta n_{1, \text { protein }} d_{\text {protein }}=(6.1 \pm 0.2) \AA \text { and } \\
\Delta n_{1, \text { surf }} d_{\text {surf }}=(1.0 \pm 0.2) \AA
\end{gathered}
$$

Because accurate values of the indices of refraction are not known for the given situation, an absolute value of the thickness cannot be determined and vice versa. However, from the combination of SHG and ellipsometry measurements, realistic estimations of $n$ and $d$ can be obtained. From polarized SHG measurements, the average tilt angle of the adsorbed surfactants was determined to be $\langle\psi\rangle \approx 44^{\circ}$. For this measurement the input polarization of the light was varied while the analyzer was in a fixed position, following the method of Heinz et al. ${ }^{17}$ Together with the known value of the stretched hydrophobic surfactant tail $(d \approx 14 \AA)$, this allows an estimation for the effective layer thickness: $d_{\text {eff }} \approx 9 \AA$. Here, the whole molecule is assumed to be linear. The index of refraction of the surfactants at $632.8 \mathrm{~nm}$ can thus be estimated to be $n_{\text {surf }}=(1.44 \pm 0.05)$, a very realistic value for this kind of molecule. When for the protein layer a refractive index between $n=1.5$ and $n=1.7$ is assumed, a value for the protein layer thickness between 17 and 37 $\AA$ is obtained, which is quite reasonable for a completed protein coverage.

For the mixed system, four possible configurations were studied in order to obtain an as complete as possible impression of the interaction behavior. A 2-fold division is made in the manner of approaching the monolayer from above or below the interface and by starting from a protein or surfactant monolayer on the clean water surface.

In the first two configurations, a stable surfactant monolayer was formed $\left(\tilde{C}_{\text {bulk }}=2 \times 10^{-6} \mathrm{M}\right)$, after which the lipase was supplied from above by a pipette at a concentration $C^{\text {pipet }}=2.2 \times 10^{-5} \mathrm{M}$ or from below by means

(17) Heinz, T. F.; Tom, H. W. K.; Shen, Y. R. Phys. Rev. A 1983, 28, 1883
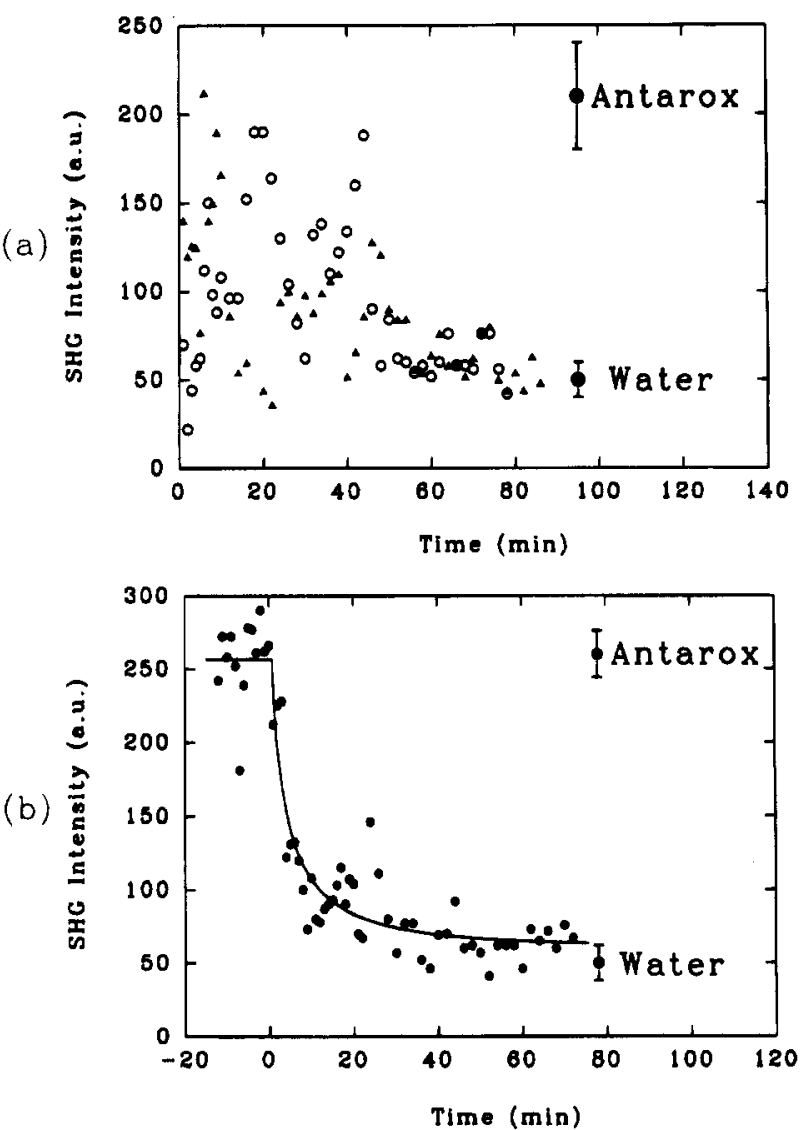

Figure 2. (a) Variation of the SHG signal after the lipase molecules are supplied from above on the Antarox monolayer system, showing the removal of the surfactant molecules from the surface for two experimental runs ( $\Delta$ and $O$ ). (b) The Antarox monolayer is removed smoothly as the protein molecules are supplied from below.

of a tube $\left(C^{\text {tube }}=3.3 \times 10^{-4} \mathrm{M}\right)$. In both cases the SHG signal level decreases from the Antarox level to the water signal level (Figure 2a,b). Therefore the proteins seem to be able to remove the surfactants from the interfacial region. The effect of the proteins in the interfacial region can thus clearly be seen by the SHG probe, though in an indirect manner. However, as we are only detecting the presence of surfactants in the SHG experiment, an alternative explanation of these observations is the binding or adsorption of the free surfactant at the protein in the bulk phase, which effectively will reduce the surfactant 

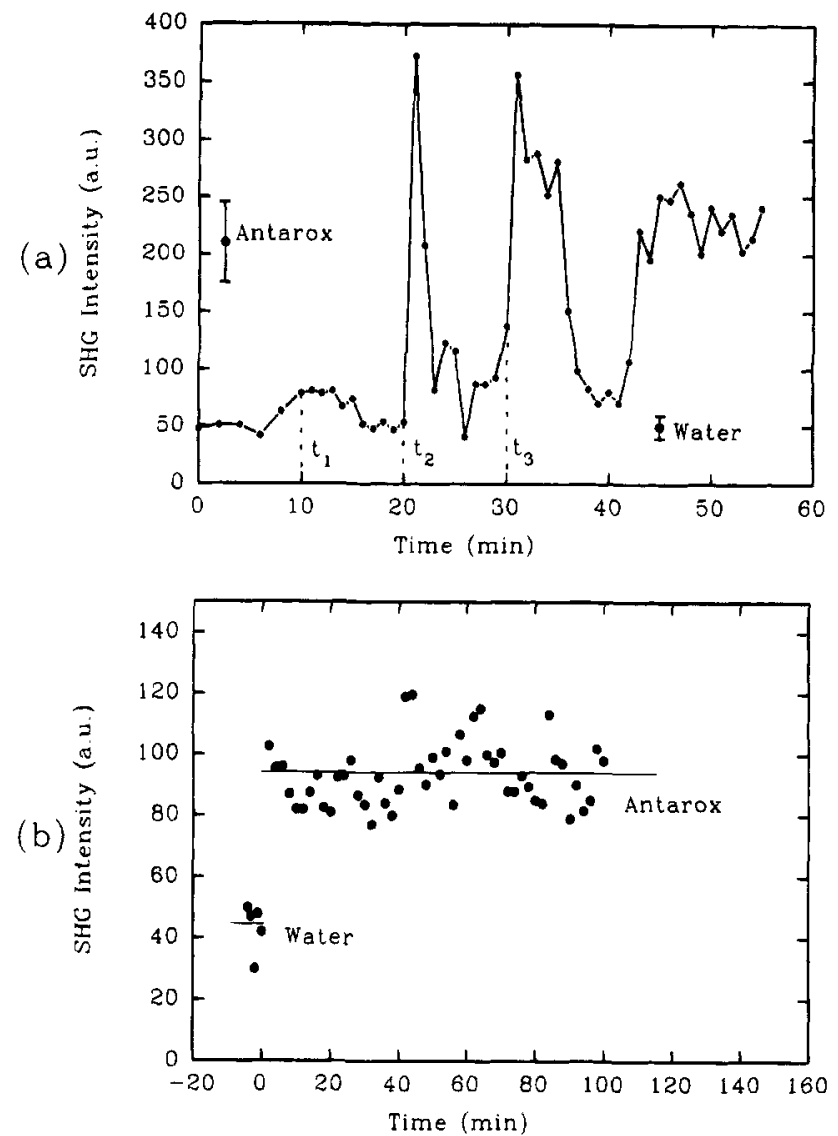

Figure 3. (a) Subsequent doses of surfactant of $5.5 \mathrm{~K}\left(t_{1}\right.$ and $t_{2}$ ) and $16.4 \mathrm{~K}\left(t_{3}\right)$ supplied from above upon the lipase layer. Only after the submission of the last dose does the Antarox reach full monolayer coverage. (b) The Antarox molecules instantaneously form a monolayer within the detection limit $(30 \mathrm{~s})$ as the surfactants are injected underneath the lipase layer at $C_{\text {bulk }}=20 \mathrm{~K}$.

bulk concentration. This will also lead to a lower surface concentration. The question remains: What is the real composition of the interface?

Therefore we did ellipsometry measurements on the system studied in Figure 2a. The first $30 \mathrm{~min} \delta \Delta$ showed fluctuations between the two values $\delta \Delta=(5.0 \pm 0.2)^{\circ}$ and $\delta \Delta=(10.0 \pm 0.2)^{\circ}$. Both surfactant and protein islands are thus present in this initial period. Thereafter the signal appeared to stabilize at $\delta \Delta=(10.0 \pm 0.2)^{\circ}$, that is, almost equal to that of a full protein monolayer. This confirms that the surfactant layer is completely removed by the forming of a protein layer.

The reversed situation was probed in two further experiments, in which the proteins are supplied first by a pipet $\left(C^{\text {pipet }}=2.2 \times 10^{-5} \mathrm{M}\right)$ and Antarox is submitted to the solution from above and from below (see Figure $3 a, b$, respectively). In the last configuration, a stronger Antarox bulk solution $(20 \mathrm{~K})$ is used to compensate for micelle formation in the bulk.

Figure 3 a shows that for the same conditions $\left(C_{\text {surfactant }}\right.$ $=5.5 \mathrm{~K}$ ) the result is consistent with Figure 2; that is, the proteins form a stable layer at the interface unaffected by the addition of surfactants from above. Even for a doublesurfactant concentration, the protein layer is not disturbed (Figure 3a, $t_{2} \rightarrow t_{3}$ ). However, by the addition of more and more Antarox (at $t_{3}$ ), the surfactants appear to remove the protein layer eventually, at a final concentration of 27 $K$. The situation is different for the configuration where $C_{\text {surfactant }}=20 \mathrm{~K}$ is supplied in the bulk in order to compensate for micelle formation. In that case, the Antarox immediately comes to the surface (see Figure

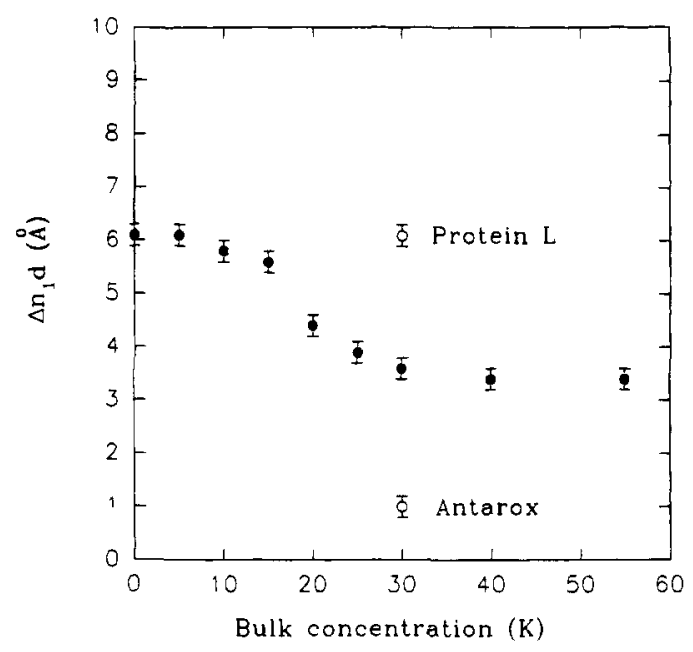

Figure 4. Ellipsometry parameter $\Delta n_{1} d$ plotted against surfactant concentration delivered to a protein aqueous solution from above, as correspondingly studied by SHG in Figure 3a. Neat water corresponds to $\Delta n_{1} d=0 \AA$. The equilibrium values have been taken $(t \approx 30 \mathrm{~min})$. Also indicated are the parameter values of the pure protein and pure Antarox solutions.

$3 b$ ), reaching in this case a final coverage of about $60 \%$. A penetration of the protein layer by the surfactant is likely to be responsible for this rapid response.

The experiment of Figure 3a was repeated by ellipsometry. Now, subsequent small doses of surfactants are supplied. Figure 4 shows the steady state variation of $\Delta n_{1} d$ as the concentration of surfactants is increased. The data shown are the final equilibrium values, obtained after the original fluctuating signal had stabilized. $\Delta n_{1} d$ is seen to diminish only substantially after a deposition of over $15 \mathrm{~K}$, in agreement with the SHG data. However, the ellipsometry signal appears to saturate intermediate of the surfactant monolayer value and the protein monolayer value, indicating an effectively mixed layer.

From Figure $3 a$, the SHG results indicate the presence of a full surfactant monolayer. Therefore a kind of double layer is formed here, in which the surfactants form a monolayer on top of a layer of proteins. The intermediate value for $\delta \Delta$ observed in ellipsometry can be the result of one of the following mechanisms. First, part of the proteins can be dissolved as a result of the increased surfactant concentration at the surface; second, the sign of the contributions to $\delta \Delta$ from the surfactant and the protein layer may be different. The latter is the case if $n_{\text {protein }}>$ $n_{\text {surfactant. }}$

In addition to the SHG data, the ellipsometry measurements also show a shift toward higher concentrations before a surfactant monolayer is formed. This suggests that the proteins force the surfactants to dissolve at low surfactant concentrations due to the micellization of the surfactants.

Also in this case (Figure 4), fluctuations are observed for low surfactant concentrations $\left(C_{\text {bulk }} \leq 20 \mathrm{~K}\right)$. The fluctuations observed for $C_{\text {bulk }}=5.0 \mathrm{~K}$ are between the two values $\delta \Delta=(4.0 \pm 0.2)^{\circ}$ and $\delta \Delta=(11.0 \pm 0.2)^{\circ}$. This is roughly equal to the fluctuations observed in case 1 (Figure $2 \mathrm{a}$ and ellipsometry), where proteins are supplied to a surfactant monolayer using the same concentrations, and indicates the coexistence of protein and surfactant domains at the surface.

In conclusion, we have studied the exchange behavior of proteins and surfactants at the water-air interface using SHG and ellipsometry as optical probes. The adsorption behavior of the surfactants as characterized by the adsorption free energy is consistent with surface 
tension measurements, though a saturated monolayer is obtained for a bulk concentration that is more than an order of magnitude lower than the cmc value. The proteins show strong surface activity. At low concentrations the surfactants dissolve and form micelles due to the presence of the proteins. An energy barrier against breaking of the surfactant micelles is observed. At sufficiently high concentrations the surfactants are able to form a monolayer on top of the proteins in the interfacial layer, despite the micellization processes. Further, in the formation of equilibrium states, surfactant and protein islands are present. This island formation is seen both in the case where the surfactant is supplied on top of a protein monolayer and in the case where proteins are delivered on top of an Antarox monolayer. Thus, combined SHG and ellipsometry application can give detailed information on the exchange processes between proteins and surfactants at the water-air interface, and about the structure of the interface thus formed.

Acknowledgment. The authors thank Dr. P. Weisenborn of Unilever Research Laboratory (Vlaardingen, The Netherlands) for supplying the surfactants and the proteins. Part of this work was supported by the "Stichting voor Fundamenteel Onderzoek der Materie" (FOM), which is financially supported by the "Nederlandse Organisatie voor Wetenschappelijk Onderzoek" (NWO). 